Communications in Physics, Vol.27, No. 1 (2017), pp. 83-89

DOI:10.15625/0868-3166/27/1/9035

\title{
RADION PRODUCTION IN HIGH ENERGY PHOTON COLLISIONS
}

\author{
BUI THI HA GIANG ${ }^{1, \dagger}$, DANG VAN SOA ${ }^{2}$ AND DAO THI LE THUY ${ }^{1}$ \\ ${ }^{1}$ Hanoi National University of Education, 136 Xuan Thuy, Hanoi, Vietnam \\ ${ }^{2}$ Hanoi Metropolitian University, 98 Duong Quang Ham, Hanoi, Vietnam \\ ${ }^{\dagger} E$-mail: giangbth@hnue.edu.vn
}

Received 20 December 2016

Accepted for publication 27 February 2017

\begin{abstract}
We study the Higgs-radion mixing and photon collisions. We consider the radion production in high energy photon collisions to search for the radion in the Randall-Sundrum model. Numerical evaluation gives observable cross-section in the future colliders.
\end{abstract}

Keywords: radion production, photon collision, Randall-Sundrum model.

Classification numbers: 12.60.-i.

\section{INTRODUCTION}

The gauge hierarchy problem is one of theoretical drawbacks of the Standard model (SM). The Randall-Sundrum (RS) model is one of many attempts to extend the SM and solve the hierarchy problem [1]. The RS setup involves two three-branes bounding a slice of 5D compact anti-de Sitter space taken to be on an $S^{1} / Z_{2}$ orbifold. Gravity is localized UV brane, while the Standard Model fields are supposed to be localized IR brane. The separation between the two 3-branes leads directly to the existence of an additional scalar called the radion $(\phi)$, corresponding to the quantum fluctuations of the distance between the two 3-branes [2]. The radion mass is considered in the range of $\mathscr{O}(10 \mathrm{GeV}) \leq m_{\phi} \leq \mathscr{O}(\mathrm{TeV})$ [3]. The common origin of the radion and Kaluza-Klein gravitons means a sensitive to brane curvature terms. The radion couples with the matter via the trace of the energy momentum tensor. Therefore, the structure of the coupling of the radion with the SM fields is similar to that of the Higgs boson. Because the Higgs search is one of the main goals of the future collider experiments, the mixing of Higgs with another particle is of major importance, if it will change the Higgs production or decay patterns. General covariance allows a possibility of mixing between the radion and the Higgs boson. The mixing of radion with Higgs will modify the Higgs and radion phenomenology significantly [4]. In Ref. [5], authors show that Higgs-dominated state at $125 \mathrm{GeV}$, where the Higgs signal is discovered by the ATLAS and CMS collaborations [6, 7], is the preferred one to the radion-dominated state at $125 \mathrm{GeV}$. Additionally, 
the associated production of Higgs bosons with the radion was also studied in Ref. [8-12].

In this paper, we study the radion production, which has been proposed as an option of photon collisions. The layout of this paper is as follows: we review the RS model in Section 2 and the mixing of the Higgs and the radion, carrying the same quantum numbers as the Higgs boson, in Section 3 mostly cited on $[1,2,12]$. Section 4 is devoted to the creation of radion and Higgs in high energy photon collisions through the $\phi, h$ propagators. Finally, we summarize our results and make conclusions in Section 5.

\section{A REVIEW OF RANDALL - SUNDRUM MODEL}

The RS model is based on a 5D spacetime with non - factorizable geometry [1]. The single extradimension is compactified on an $S^{1} / Z_{2}$ orbifold of which two fixed points accommodate two three-branes (4D hyper-surfaces): the UV brane and the IR brane. $S^{1}$ is a sphere in one dimension and $Z_{2}$ is the multiplicative group $\{-1,1\}$. The fundamental action is

$$
S=S_{H}+S_{M}=\int d^{4} x \int_{-\pi}^{\pi} d \phi \sqrt{-G}\left(2 M_{5}^{3} R-\Lambda\right),
$$

where $M_{5}$ is the fundamental 5D mass scale, $\mathrm{R}$ is the 5D Ricci scalar and $\mathrm{G}$ is the determinant of the five-dimensional metric, $\Lambda$ is the $5 \mathrm{D}$ cosmological constant [2].

The background metric reads:

$$
d s^{2}=e^{-2 k r_{c}|\phi|} \eta_{\mu \nu} d x^{\mu} d x^{\nu}+r_{c}^{2} d \phi^{2} \quad-\pi \leq \phi \leq \pi,
$$

with $x^{\mu}(\mu=0,1,2,3)$ the coordinates on the 4D hyper-surfaces of constant $\phi, r_{c}$ the compactification radius and $k$ the bulk curvature. The four dimensional effective action is obtained by integrating out the extra dimension.

The Higgs action can be shown as

$$
S_{H}=\int d^{4} x\left[\eta^{\mu v} D_{\mu} \widetilde{H}^{+} D_{v} \widetilde{H}-\lambda\left(\widetilde{H}^{+} \widetilde{H}-\left(e^{-k r_{c} \pi} v\right)^{2}\right)^{2}\right],
$$

where $v$ is a mass parameter, the Higgs field $H=e^{k r_{c} \pi} \widetilde{H}$.

The physical Higgs mass can be written

$$
m=e^{-k r_{c} \pi} m_{0},
$$

where $m_{0}$ is of Planck scale. If the value of $m_{0}$ is of the order of $10^{19} \mathrm{GeV}, m \simeq 1 \mathrm{TeV}$. The physical mass is warped down to the weak scale. Since the scale of weak interactions $M_{W} \simeq 10^{-16} M_{P l}$, the applicable value for size of the extra dimension is assessed by

$$
k r_{c} \pi \simeq 35 .
$$

The compactification radius, $r_{c}$ is arbitrary and is treated as a free parameter in the theory. Consequently, the hierarchy problem is addressed.

When the backreaction of the metric background due to the scalar field is small, the wavefunction is

$$
F(x, \phi)=\frac{\varphi(x)}{\Lambda_{\phi}} e^{2 k r_{c}(\phi-\pi)},
$$


where $\Lambda_{\phi}=\sqrt{6} M_{P l} e^{-k r_{c} \pi}$ is the vacuum expectation value (VEV) of the radion. The mass of radion depends on the mass of the bulk scalar and can be smaller than $1 \mathrm{TeV}$ [2].

The gravity-scalar mixing is described by the following action

$$
S_{\xi}=\xi \int d^{4} x \sqrt{g_{v i s}} R\left(g_{\text {vis }}\right) \hat{H}^{+} \hat{H},
$$

where $\xi$ is the mixing parameter [13-16], $R\left(g_{v i s}\right)$ is the Ricci scalar for the metric $g_{v i s}^{\mu v}=\Omega_{b}^{2}(x)\left(\eta^{\mu v}+\right.$ $\left.\varepsilon h^{\mu v}\right)$ induced on the visible brane, $\Omega_{b}(x)=e^{-k r_{c} \pi}\left(1+\frac{\phi_{0}}{\Lambda_{\phi}}\right)$ is the warp factor, $\phi_{0}$ is the canonically normalized massless radion field, $\hat{H}$ is the Higgs field in the 5D context before rescaling to canonical normalization on the brane. With $\xi \neq 0$, there is neither a pure Higgs boson nor pure radion mass eigenstate. There are four independent parameters $\Lambda_{\phi}, m_{h}, m_{\phi}, \xi$ that must be specified to fix the state mixing parameters. We consider the case of $\Lambda_{\phi}=5 \mathrm{TeV}$ and $\frac{m_{0}}{M_{P}}=0.1$, which makes the radion stabilization model most natural [17].

\section{THE MIXING OF THE HIGGS BOSON AND THE RADION}

The radion coupling to the gauge boson differs from the coupling of the gauge bosons to the Higgs. In case of massless gauge bosons, along with the tree level coupling, the effects of the localized trace anomalies on the $\mathrm{TeV}$ brane are also included, and these are proportional to the $\beta$-function coefficient of the light fields localized on the $\mathrm{TeV}$ brane $[18,19]$. The interaction terms of the Higgs and the radion with gamma couplings in the mixing scenario are given by [2]

$$
\begin{aligned}
\mathscr{L}_{h}^{\gamma \gamma}= & \frac{h}{4 v}\left\{\left[a \frac{\alpha_{s}}{2 \pi} b_{Q C D}^{h}+\gamma c\left(\frac{1}{k r_{c} \pi}+\frac{\alpha_{s}}{2 \pi} b_{Q C D}^{R}\right)\right] G_{\mu \nu}^{a} G^{a \mu v}\right. \\
& \left.+\left[a \frac{\alpha}{2 \pi} b_{E M}^{h}+\gamma c\left(\frac{1}{k r_{c} \pi}+\frac{\alpha}{2 \pi} b_{E M}^{R}\right)\right] F_{\mu v} F^{\mu v}\right\}, \\
\mathscr{L}_{\phi}^{\gamma \gamma}= & \frac{\phi}{4 v}\left\{\left[b \frac{\alpha_{s}}{2 \pi} b_{Q C D}^{h}+\gamma d\left(\frac{1}{k r_{c} \pi}+\frac{\alpha_{s}}{2 \pi} b_{Q C D}^{R}\right)\right] G_{\mu \nu}^{a} G^{a \mu v}\right. \\
& \left.+\left[b \frac{\alpha}{2 \pi} b_{E M}^{h}+\gamma d\left(\frac{1}{k r_{c} \pi}+\frac{\alpha}{2 \pi} b_{E M}^{R}\right)\right] F_{\mu \nu} F^{\mu v}\right\},
\end{aligned}
$$

where $\alpha_{s}$ and $\alpha$ are the strong and the electromagnetic coupling constants, $G, F$ are the gluon and the photon field functions, respectively. The parameters $a, b, c, d$ define the mixing between the $\xi=0$ states and the $\xi \neq 0$ mass eigenstates [12]

$$
\begin{aligned}
& a=\cos \theta+\frac{6 \xi \gamma}{Z} \sin \theta, b=\sin \theta-\frac{6 \xi \gamma}{Z} \cos \theta, c=\frac{-\sin \theta}{Z}, d=\frac{\cos \theta}{Z}, \\
& b_{Q C D}^{h}=\tau_{f}\left(1+\left(1-\tau_{f}\right) f\left(\tau_{f}\right)\right), \\
& b_{E M}^{h}=\frac{8}{3}\left[\tau_{\tau}\left(1+\left(1-\tau_{f}\right) f\left(\tau_{f}\right)\right)\right]-\left[2+3 \tau_{W}+3 \tau_{W}\left(2-\tau_{W}\right) f\left(\tau_{W}\right)\right], \\
& b_{Q C D}^{R}=7+\tau_{f}\left(1+\left(1-\tau_{f}\right) f\left(\tau_{f}\right)\right), \\
& b_{E M}^{R}=-\frac{11}{3}+\frac{8}{3}\left[\tau_{\tau}\left(1+\left(1-\tau_{f}\right) f\left(\tau_{f}\right)\right)\right]-\left[2+3 \tau_{W}+3 \tau_{W}\left(2-\tau_{W}\right) f\left(\tau_{W}\right)\right],
\end{aligned}
$$




$$
\begin{aligned}
& f(\tau)=\left(\sin ^{-1} \frac{1}{\sqrt{\tau}}\right)^{2}(\text { for } \tau>1), \\
& f(\tau)=-\frac{1}{4}\left(\log \frac{\eta_{+}}{\eta_{-}}-i \pi\right)^{2}(\text { for } \tau<1), \\
& \eta_{ \pm}=1 \pm \sqrt{1-\tau}, \tau_{i}=\left(\frac{2 m_{i}}{m_{s}}\right)^{2} .
\end{aligned}
$$

$m_{i}$ is the mass of the internal loop particle and $m_{s}$ is the mass of the scalar state, $h$ or $\phi$. Here, $\tau_{f}=$ $\left(\frac{2 m_{f}}{m_{s}}\right)^{2}, \tau_{W}=\left(\frac{2 m_{W}}{m_{s}}\right)^{2}$ denote the squares of fermion and $\mathrm{W}$ gauge boson mass ratios, respectively. $Z^{2}=1+6 \gamma^{2} \xi(1-6 \xi)=\beta-36 \xi^{2} \gamma^{2}$ is the coefficient of the radion kinetic term after undoing the kinetic mixing, $\gamma=v / \Lambda_{\phi}, v=246 \mathrm{GeV}$. The mixing angle $\theta$ is

$$
\tan 2 \theta=12 \gamma \xi Z \frac{m_{h_{0}}^{2}}{m_{h_{0}}^{2}\left(Z^{2}-36 \xi^{2} \gamma^{2}\right)-m_{\phi_{0}}^{2}},
$$

where $m_{h_{0}}$ and $m_{\phi_{0}}$ are the Higgs and radion masses before mixing. The couplings for two Higgs bosons with radion are given by

$$
\begin{aligned}
\mathscr{L}_{\phi}^{h h}= & \frac{\phi}{\Lambda_{\phi}}\left\{\left[-a^{2} d\left(\partial_{\mu} h \partial^{\mu} h+2 m_{h}^{2} h h\right)\right]+4 a b c m_{h}^{2} h h\right\} \\
& -3 \frac{m_{h}^{2}}{2 v}\left(a^{2} b \phi h^{2}\right)-\frac{3 \xi}{\Lambda_{\phi}}\left[\left(a^{2} d h^{2} \square \phi+2 a b c h \square h \phi\right)\right] \\
& -6 \xi \frac{v}{\Lambda_{\phi}}\left[\left(a c d h^{2} \square \phi+(a d+b c) c h \square h \phi\right)\right] .
\end{aligned}
$$

\section{RADION PRODUCTION IN PHOTON COLLISIONS}

\section{IV.1. The scattering amplitude}

In this section, we consider the collision process in which the initial state contains $\gamma$ coupling, the final state contains the couple of radions or a pair of radion and Higgs boson through $\phi, h$ propagator

$$
\gamma\left(p_{1}\right)+\gamma\left(p_{2}\right) \rightarrow \phi\left(k_{1}\right)+X\left(k_{2}\right) .
$$

Here $p_{i}, k_{i}(\mathrm{i}=1,2)$ stand for the momentums, $\mathrm{X}$ stands for the radion or Higgs. There are three Feynman diagrams contributing to reaction (20), representing the $s, u, t$-channels exchange depicted in Fig. 1.

We obtain the results in the $s, u, t$-channels

$$
\begin{aligned}
& M_{s}=-\left[C_{\gamma \phi} \frac{\bar{g}_{\phi \phi X}}{q_{s}^{2}-m_{\phi}^{2}}+C_{\gamma h} \frac{\bar{g}_{\phi h X}}{q_{s}^{2}-m_{h}^{2}}\right] \varepsilon_{\alpha}\left(p_{1}\right)\left[\left(p_{1} p_{2}\right) \eta^{\alpha \beta}-p_{1}^{\beta} p_{2}^{\alpha}\right] \varepsilon_{\beta}\left(p_{2}\right), \\
& M_{u}=i C_{\gamma \phi} C_{\gamma X} \frac{1}{q_{u}^{2}}\left[\left(p_{2} q_{u}\right) \eta_{\rho}^{v}-p_{2 \rho} q_{u}^{v}\right] \varepsilon_{v}\left(p_{2}\right)\left[\left(p_{1} q_{u}\right) \eta^{\mu \rho}-p_{1}^{\rho} q_{u}^{\mu}\right] \varepsilon_{\mu}\left(p_{1}\right), \\
& M_{t}=i C_{\gamma \phi} C_{\gamma X} \frac{1}{q_{t}^{2}}\left[\left(p_{1} q_{t}\right) \eta_{\sigma}^{\mu}-p_{1 \sigma} q_{t}^{\mu}\right] \varepsilon_{\mu}\left(p_{1}\right)\left[\left(p_{2} q_{t}\right) \eta^{v \sigma}-p_{2}^{\sigma} q_{t}^{v}\right] \varepsilon_{v}\left(p_{2}\right) .
\end{aligned}
$$


where $C_{\gamma \phi}, C_{\gamma h}, \bar{g}_{\phi \phi h}, \bar{g}_{\phi \phi \phi}, \bar{g}_{\phi h h}, \bar{g}_{\phi h \phi}$ are given by [12], $q_{s}=p_{1}+p_{2}=k_{1}+k_{2}, q_{u}=p_{1}-k_{2}=$ $k_{1}-p_{2}, q_{t}=p_{1}-k_{1}=k_{2}-p_{2}$ and $s=\left(p_{1}+p_{2}\right)^{2}$ is the square of the collision energy.
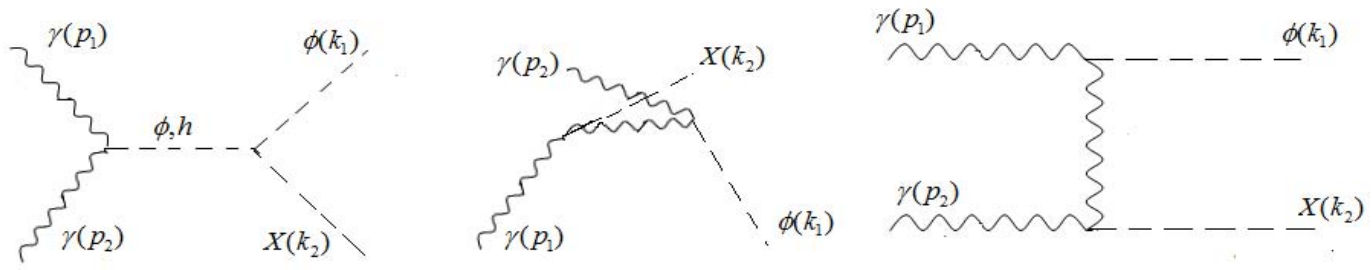

Fig. 1. Feynman diagrams for $\gamma \gamma \rightarrow \phi X$. X stands for the radion or Higgs.

\section{IV.2. The differential and total cross sections}

From the expressions of the differential cross-section [20]

$$
\frac{d \sigma}{d(\cos \psi)}=\frac{1}{32 \pi s} \frac{|\vec{k}|}{|\vec{p}|}\left|M_{f i}\right|^{2},
$$

where $M_{f i}$ is the scattering amplitude, we assess the number and make the identification, evaluation of the results obtained from the dependence of the differential cross-section $d \sigma$ by $\cos \psi$ ( $\psi$-the angle between momenta of the initial photon and the final radion), the total cross-section $\sigma$ fully follows the collision energy $\sqrt{s}$.

We choose $m_{h}=125 \mathrm{GeV}$ (CMS), $\Lambda_{\phi}=5 \mathrm{TeV}, \xi=1 / 6$ [12], $m_{\phi}=110 \mathrm{GeV}$ [2]. We give some estimates for the cross-sections as follows:
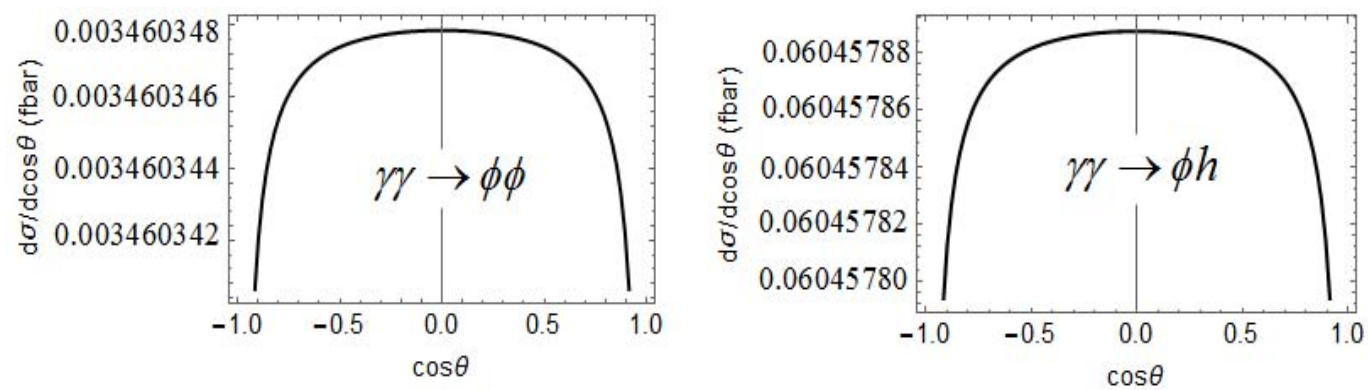

Fig. 2. The differential cross-section in photon collisions as a function of $\cos \psi$ at $\sqrt{s}=3 \mathrm{TeV}$.

i) In Fig. 2, we plot the differential cross-section as the function of $\cos \psi$ at the fixed collision energy, $\sqrt{s}=3 \mathrm{TeV}$ (CLIC). We show that the differential cross-sections are nearly unchanged when $\cos \psi$ changes from -1 to 1 . The ability of search the radion is nearly the same to every direction. However, the differential cross section in $\gamma \gamma \rightarrow \phi h$ is about 17 times as large as that in $\gamma \gamma \rightarrow \phi \phi$. 
ii) In Fig. 3, we plot the total cross-section as a function of the collision energy $\sqrt{s}$. We show that the total cross-section increases gradually in the region $1 \mathrm{TeV}<\sqrt{\mathrm{s}}<5 \mathrm{TeV}$. The total cross section in $\gamma \gamma \rightarrow \phi h$ is also about 17 times as large as that in $\gamma \gamma \rightarrow \phi \phi$.
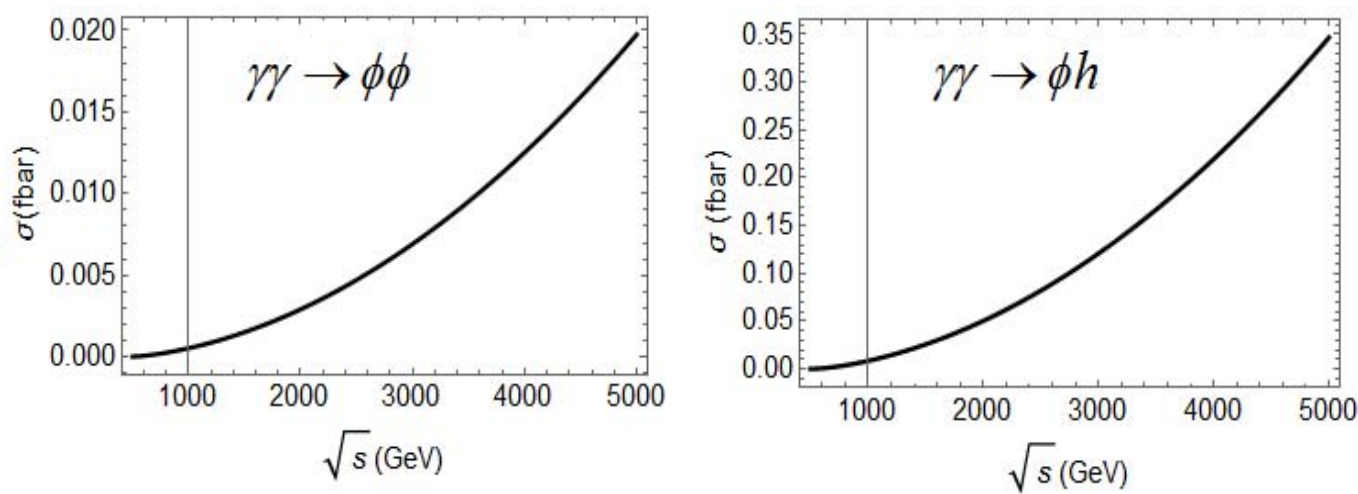

Fig. 3. The total cross-section in photon collisions as a function of the collision energy $\sqrt{s}$.

\section{CONCLUSION}

In this paper, we have evaluated the radion production in the RS model at the high energy photon - photon colliders. The result shows that cross-sections of radion - Higgs are much bigger than that pair production of radion in the same conditions. Additionally, the ability of search the radion is nearly the same to every direction.

\section{ACKNOWLEDGEMENT}

The work is supported in part by the National Foundation for Science and Technology Development (NAFOSTED) of Vietnam under Grant No. 103.01-2016.44.

\section{REFERENCES}

[1] L. Randall and R. Sundrum, Phys. Rev. Lett. 83 (1999) 3370.

L. Randall and R. Sundrum, Phys. Rev. Lett. 83 (1999) 4690.

[2] M. Frank, K. Huitu, U. Maitra, M. Patra, Phys. Rev. D 94, (2016) 055016.

[3] G.D. Kribs, eConf C010630 (2001) P317.

[4] M. Chaichian, A. Datta, K. Huitu and Z. Yua, Phys.Lett. B 524 (2002) 161-169.

[5] E. E. Boos, V. E. Bunichev, M. A. Perfilov, M. N. Smolyakov, and I. P. Volobuev Phys. Rev. D 92 (2015) 095010.

[6] G. Aad et al., ATLAS Collaboration,Phys. Lett. B 716 (2012) 1-29.

[7] S. Chatrchyan et al., CMS Collaboration, Phys. Lett. B 716 (2012) 30-61.

[8] K. Cheung, C. S. Kim and J-H. Song, Phys. Rev. D 67 (2003) 075017.

[9] K. Cheung, C. S. Kim and J-H. Song, Phys. Rev. D 72 (2005) 115015.

[10] H. Davoudiasl, T. McElmurry and A. Soni, Phys. Rev. D 82 (2010) 115028.

[11] V. P. Goncalves and W. K. Sauter, Phys. Rev. D 82 (2010) 056009.

[12] D. Dominici, B. Grzadkowski, J. F. Gunion and M. Toharia, Nucl. Phys. B 671 (2003) 243.

[13] W. D. Goldberger and M. B. Wise, Phys. Rev. Lett. 83 (1999) 4922, arXiv:hep-ph/9907447. 
[14] S. A. Li, C. S. Li, H. T. Li, J. Gao, Phys. Rev. D91 (2015) 014027, arxiv:hep-ph/1408.2762.

[15] J. J. van der Bij, Acta Phys. Polon. D 25 (1994) 827.

[16] R. Raczka, M. Pawlowski, Found. Phys. 24 (1994) 1305.

[17] H. Davoudiasl, J. L. Hewett and T. G. Rizzo, Phys. Rev. D 63 (2001) 075004.

[18] C. Csaki, M. L. Graesser and G. D. Kribs, Phys. Rev. D 63 (2001) 065002.

[19] C. Csaki, J. Hubisz and S. J. Lee, Phys. Rev. D 76 (2007) 125015.

[20] M. E. Peskin and D. V. Schroeder, An Introduction to Quantum Field Theory, Addision-Wesley Publishing, 1995. 